hep-th/0601228

\title{
BTZ Black Hole with Chern-Simons and Higher Derivative Terms
}

\author{
Bindusar Sahoo and Ashoke Sen \\ Harish-Chandra Research Institute \\ Chhatnag Road, Jhusi, Allahabad 211019, INDIA \\ E-mail: bindusar@mri.ernet.in, ashoke.sen@cern.ch, sen@mri.ernet.in
}

\begin{abstract}
The entropy of a BTZ black hole in the presence of gravitational Chern-Simons terms has previously been analyzed using Euclidean action formalism. In this paper we treat the BTZ solution as a two dimensional black hole by regarding the angular coordinate as a compact direction, and use Wald's Noether charge method to calculate the entropy of this black hole in the presence of higher derivative and gravitational Chern-Simons terms. The parameters labelling the black hole solution can be determined by extremizing an entropy function whose value at the extremum gives the entropy of the black hole.
\end{abstract}




\section{Contents}

1 Introduction $\quad 2$

2 The Two Dimensional View $\quad 3$

3 Extremal BTZ Black Holes $\quad 5$

4 Non-extremal BTZ Black Holes $\quad 8$

\section{Introduction}

BTZ solution describes a black hole in three dimensional theory of gravity with negative cosmological constant[1] and often appears as a factor in the near horizon geometry of higher dimensional black holes in string theory[2]. For this reason it has provided us with a useful tool for relating black hole entropy to the degeneracy of microstates of the black hole, both in three dimensional theories of gravity and also in string theory[2, 3]. Initial studies involved relating the Bekenstein-Hawking formula for BTZ black hole entropy in two derivative theories of gravity to the Cardy formula for the degeneracy of states in the two dimensional conformal field theory living on the asymptotic boundary. Later this was generalized to higher derivative theories of gravity, where the lagrangian density contains arbitrary powers of Riemann tensor and its covariant derivatives[4]. For computing entropy of black holes in such theories one can no longer use the area formula. Instead one needs to use the Noether charge method developed by Wald[5, 6, 7, 8].

In three dimensions one can also add to the action the gravitational Chern-Simons terms. In this case the Lagrangian density cannot be written in a manifestly covariant form and as a result Wald's formalism cannot be applied in a straightforward fashion. For this reason the effect of this term on the black hole entropy was analyzed in $[9,10]$ using the Euclidean action formalism[11]. A different Euclidean method yielding the same result can be found in [12].

The goal of this paper is to compute the entropy of BTZ black holes in the presence of Chern-Simons and higher derivative terms using Wald's Noether charge method. In order to do this we regard the BTZ black hole as a two dimensional configuration by treating the angular coordinate as a compact direction[13]. The black hole entropy is then calculated using the dimensionally reduced two dimensional theory. This has the advantage that the 
Chern-Simons term, which was not manifestly covariant in three dimensions, reduces to a manifestly covariant set of terms in two dimensions[14]. Hence Wald's formula for the black hole entropy can be applied in a straightforward fashion. The result agrees with the one calculated using the Euclidean action formalism.

The rest of the paper is organized as follows. In section 2 we discuss the dimensional reduction of a general three dimensional theory of gravity, including the gravitational Chern-Simons term, to two dimensions and describe the BTZ solution from two dimensional viewpoint. In section 3 we calculate the entropy of extremal BTZ black holes using the entropy function formalism[15] which is known to be equivalent to Wald's Noether charge method. In section 4 we calculate the entropy of a non-extremal BTZ black hole using Wald's method directly. Both for the extremal and the non-extremal black holes the parameters labelling the solution can be obtained by extremizing an entropy function whose value at the extremum gives the entropy.

\section{The Two Dimensional View}

Let us consider a three dimensional theory of gravity with metric $G_{M N}(0 \leq M, N \leq 2)$ and a general action of the form: ${ }^{1}$

$$
S=\int d^{3} x \sqrt{-\operatorname{det} G}\left[\mathcal{L}_{0}^{(3)}+\mathcal{L}_{1}^{(3)}\right]
$$

Here $\mathcal{L}_{0}^{(3)}$ denotes an arbitrary scalar constructed out of the metric, the Riemann tensor and covariant derivatives of the Riemann tensor. On the other hand $\sqrt{-\operatorname{det} G} \mathcal{L}_{1}^{(3)}$ denotes the gravitational Chern-Simons term:

$$
\sqrt{-\operatorname{det} G} \mathcal{L}_{1}^{(3)}=K \Omega_{3}(\widehat{\Gamma})
$$

where $K$ is a constant, $\widehat{\Gamma}$ is the Christoffel connection constructed out of the metric $G_{M N}$ and

$$
\Omega_{3}(\widehat{\Gamma})=\epsilon^{M N P}\left[\frac{1}{2} \widehat{\Gamma}_{M S}^{R} \partial_{N} \widehat{\Gamma}_{P R}^{S}+\frac{1}{3} \widehat{\Gamma}_{M S}^{R} \widehat{\Gamma}_{N T}^{S} \widehat{\Gamma}_{P R}^{T}\right]
$$

$\epsilon$ is the totally anti-symmetric symbol with $\epsilon^{012}=1$.

\footnotetext{
${ }^{1}$ We could add any number of scalar fields without changing the final result since they must be frozen to constant values in order to comply with the homogeneity of the BTZ configuration.
} 
We shall consider field configurations where one of the coordinates (say $y \equiv x^{2}$ ) is compact with period $2 \pi$ and the metric is independent of this compact direction. In this case we can define two dimensional fields through the relation:

$$
G_{M N} d x^{M} d x^{N}=\phi\left[g_{\mu \nu} d x^{\mu} d x^{\nu}+\left(d y+A_{\mu} d x^{\mu}\right)^{2}\right] .
$$

Here $g_{\mu \nu}(0 \leq \mu, \nu \leq 1)$ denotes a two dimensional metric, $A_{\mu}$ denotes a two dimensional gauge field and $\phi$ denotes a two dimensional scalar field. In terms of these two dimensional fields the action takes the form:

$$
S=\int d^{2} x \sqrt{-\operatorname{det} g}\left[\mathcal{L}_{0}^{(2)}+\mathcal{L}_{1}^{(2)}\right]
$$

where

$$
\sqrt{-\operatorname{det} g} \mathcal{L}_{0}^{(2)}=\int d y \sqrt{-\operatorname{det} G} \mathcal{L}_{0}^{(3)}=2 \pi \sqrt{-\operatorname{det} G} \mathcal{L}_{0}^{(3)},
$$

and[14]

$$
\sqrt{-\operatorname{det} g} \mathcal{L}_{1}^{(2)}=K \pi\left[\frac{1}{2} R \varepsilon^{\mu \nu} F_{\mu \nu}+\frac{1}{2} \varepsilon^{\mu \nu} F_{\mu \tau} F^{\tau \sigma} F_{\sigma \nu}\right] .
$$

Here $R$ is the scalar curvature of the two dimensional metric $g_{\mu \nu}$ :

$$
\begin{aligned}
& \Gamma_{\nu \rho}^{\mu}=\frac{1}{2} g^{\mu \sigma}\left(\partial_{\nu} g_{\sigma \rho}+\partial_{\rho} g_{\sigma \nu}-\partial_{\sigma} g_{\nu \rho}\right) \\
& R_{\nu \rho \sigma}^{\mu}=\partial_{\rho} \Gamma_{\nu \sigma}^{\mu}-\partial_{\sigma} \Gamma_{\nu \rho}^{\mu}+\Gamma_{\tau \rho}^{\mu} \Gamma_{\nu \sigma}^{\tau}-\Gamma_{\tau \sigma}^{\mu} \Gamma_{\nu \rho}^{\tau} \\
& R_{\nu \sigma}=R_{\nu \mu \sigma}^{\mu}, \quad R=g^{\nu \sigma} R_{\nu \sigma},
\end{aligned}
$$

$\varepsilon^{\mu \nu}$ is the totally antisymmetric symbol with $\varepsilon^{01}=1$, and

$$
F_{\mu \nu}=\partial_{\mu} A_{\nu}-\partial_{\nu} A_{\mu}
$$

(2.6) follows in a straightforward fashion from (2.1). (2.7) comes from dimensional reduction of the Chern-Simons term after throwing away total derivative terms and was worked out in [14]. Note that although the Chern-Simons term cannot be expressed in a manifestly covariant form in three dimensions, it does reduce to a manifestly covariant expression in two dimensions.

A general BTZ black hole in the three dimensional theory is described by the metric:

$$
G_{M N} d x^{M} d x^{N}=-\frac{\left(\rho^{2}-\rho_{+}^{2}\right)\left(\rho^{2}-\rho_{-}^{2}\right)}{l^{2} \rho^{2}} d \tau^{2}+\frac{l^{2} \rho^{2}}{\left(\rho^{2}-\rho_{+}^{2}\right)\left(\rho^{2}-\rho_{-}^{2}\right)} d \rho^{2}+\rho^{2}\left(d y-\frac{\rho_{+} \rho_{-}}{l \rho^{2}} d \tau\right)^{2},
$$


where $l, \rho_{+}$and $\rho_{-}$are parameters labelling the solution. ${ }^{2}$ Comparing this with (2.4) gives[13]

$$
\begin{aligned}
& \phi=\rho^{2}, \quad A_{\mu} d x^{\mu}=-\frac{\rho_{+} \rho_{-}}{l \rho^{2}} d \tau \\
& g_{\mu \nu} d x^{\mu} d x^{\nu}=-\frac{\left(\rho^{2}-\rho_{+}^{2}\right)\left(\rho^{2}-\rho_{-}^{2}\right)}{l^{2} \rho^{4}} d \tau^{2}+\frac{l^{2}}{\left(\rho^{2}-\rho_{+}^{2}\right)\left(\rho^{2}-\rho_{-}^{2}\right)} d \rho^{2} .
\end{aligned}
$$

\section{Extremal BTZ Black Holes}

We shall define a general extremal black hole in the two dimensional theory to be the one whose near horizon geometry is $A d S_{2}$ and for which the scalar field $\phi$ and the gauge field strength $F_{\mu \nu}$ are invariant under the $S O(2,1)$ isometry of the $A d S_{2}$ background[15]. The most general near horizon background consistent with this requirement is

$$
g_{\mu \nu} d x^{\mu} d x^{\nu}=v\left(-r^{2} d t^{2}+\frac{d r^{2}}{r^{2}}\right), \quad F_{r t}=e, \quad \phi=u
$$

where $v, e$ and $u$ are constants. Following $[15,16]$ we define

$$
f(u, v, e)=\sqrt{-\operatorname{det} g}\left(\mathcal{L}_{0}^{(2)}+\mathcal{L}_{1}^{(2)}\right)
$$

evaluated in the background (3.1), and

$$
\mathcal{E}(u, v, e, q)=2 \pi(e q-f(u, v, e))
$$

As was shown in [15], the near horizon values of $u, v$ and $e$ for an extremal black hole with electric charge $q$ is obtained by extremizing the 'entropy function' $\mathcal{E}$ with respect to these variables. Furthermore, Wald's entropy for this black hole is given by the value of the function $\mathcal{E}$ at this extremum[15].

Using eqs.(2.6), (2.7) and (3.1), (3.2) we see that for the theory considered here,

$$
f(u, v, e)=f_{0}(u, v, e)+\pi K\left(2 e v^{-1}-e^{3} v^{-2}\right),
$$

where

$$
f_{0}(u, v, e)=2 \pi \sqrt{-\operatorname{det} G} \mathcal{L}_{0}^{(3)} .
$$

\footnotetext{
${ }^{2}$ Since the local geometry of a BTZ black hole is that of $A d S_{3}$ which is maximally symmetric, the higher derivative corrections do not change the structure of the solution. However for a black hole carrying a given mass and angular momentum, the values of the parameters $l, \rho_{+}$and $\rho_{-}$depend on the higher derivative terms.
} 
Let us now specialize to the case of extremal BTZ black holes. These correspond to choosing $\rho_{-}= \pm \rho_{+}$in (2.10), (2.11) and the near horizon limit is obtained by taking $\rho$ close to $\rho_{+}$. Defining

$$
r=\rho-\rho_{+}, \quad t=\frac{4}{l^{2}} \tau,
$$

we can express (2.10) (2.11) for $\rho_{-}= \pm \rho_{+}$and small $r$ as

$$
\begin{gathered}
G_{M N} d x^{M} d x^{N}=\frac{l^{2}}{4}\left(-r^{2} d t^{2}+\frac{d r^{2}}{r^{2}}\right)+\rho_{+}^{2}\left(d y \pm\left(-\frac{l}{4}+\frac{l}{2 \rho_{+}} r\right) d t\right)^{2} \\
\phi=\rho_{+}^{2}, \quad A_{\mu} d x^{\mu}= \pm\left(-\frac{l}{4}+\frac{l}{2 \rho_{+}} r\right) d t, \quad g_{\mu \nu} d x^{\mu} d x^{\nu}=\frac{l^{2}}{4 \rho_{+}^{2}}\left(-r^{2} d t^{2}+\frac{d r^{2}}{r^{2}}\right) .
\end{gathered}
$$

Comparison with (3.1) now yields

$$
u=\rho_{+}^{2}, \quad v=\frac{l^{2}}{4 \rho_{+}^{2}}, \quad e= \pm \frac{l}{2 \rho_{+}} .
$$

Note that instead of three independent parameters $u, v$ and $e$, we now have two independent parameters $l$ and $\rho^{+}$labelling the near horizon geometry. In particular $v$ and $e$ satisfy the relation

$$
v=e^{2}
$$

This is a reflection of the fact that the BTZ black hole is locally $A d S_{3}$ and hence has a higher degree of symmetry than the more general configuration considered in (3.1). This is a consistent truncation of the parameter space and hence we can extremize the entropy function $\mathcal{E}$ subject to this constraint. We shall choose $e$ and

$$
l=2 \sqrt{u e^{2}}
$$

as independent variables.

Our next step will be to investigate the structure of $f_{0}$ given in (3.5) for the extremal BTZ black hole solution described above. Since the BTZ black hole is locally the maximally symmetric $A d S_{3}$ space, $\mathcal{L}_{0}^{(3)}$, being a scalar constructed out of the Riemann tensor and its covariant derivatives, must be a constant. Furthermore since locally BTZ metrics for different values of $\rho_{ \pm}$are related by coordinate transformation, $\mathcal{L}_{0}^{(3)}$ must be independent of $\rho_{ \pm}$and hence is a function of $l$ only. Let us define

$$
h(l)=\mathcal{L}_{0}^{(3)}
$$


evaluated in the BTZ black hole geometry. (Note that this definition is independent of whether we are using the extremal or non-extremal metric.) Since for the extremal black hole metric (3.7)

$$
\sqrt{-\operatorname{det} G}=\frac{l^{2} \rho_{+}}{4}=\frac{l^{3}}{8|e|},
$$

we get

$$
f_{0}=2 \pi \sqrt{-\operatorname{det} G} \mathcal{L}_{0}^{(3)}=\frac{1}{|e|} g(l)
$$

where

$$
g(l)=\frac{\pi l^{3} h(l)}{4} .
$$

Eqs.(3.3), (3.4), (3.10) and (3.14) now give

$$
\mathcal{E}=2 \pi\left(q e-\frac{1}{|e|} g(l)-\frac{\pi K}{e}\right) .
$$

We need to extremize this with respect to $l$ and $e$. The extremization with respect to $l$ requires extremization of $g(l)$ with respect to $l$. Let us define

$$
C=-\frac{1}{\pi} g(l)
$$

at the extremum of $g$. This gives

$$
\mathcal{E}=2 \pi\left(q e+\frac{\pi C}{|e|}-\frac{\pi K}{e}\right) .
$$

We shall assume that $C \geq|K|$. Extremizing (3.18) with respect to $e$ we now get:

$$
\begin{aligned}
e & =\sqrt{\frac{\pi(C-K)}{q}} \quad \text { for } q>0, \\
& =\sqrt{\frac{\pi(C+K)}{|q|}} \quad \text { for } q<0 .
\end{aligned}
$$

Furthermore, at the extremum,

$$
\begin{aligned}
\mathcal{E} & =2 \pi \sqrt{\frac{c_{R} q}{6}} \quad \text { for } q>0 \\
& =2 \pi \sqrt{\frac{c_{L}|q|}{6}} \quad \text { for } q<0
\end{aligned}
$$

where we have defined

$$
c_{L}=24 \pi(C+K), \quad c_{R}=24 \pi(C-K) .
$$


(3.20) gives the entropy of extremal BTZ black hole. Since the conserved charge $q$ measures momemtum along $y$, which for a BTZ black hole represents the angular momentum $J$, eqs.(3.20), (3.21) are in agreement with the results of [9] for extremal BTZ black holes with mass $=|J|$.

Note that the Chern-Simons term plays no role in the determination of the parameter $l$ and

$$
c_{L}+c_{R}=48 \pi C=-48 g(l) .
$$

This is a reflection of the fact that in three dimensions the effect of the Chern-Simons term on the equations of motion involves covariant derivative of the Ricci tensor[14] which vanishes for BTZ solution. On the other hand

$$
c_{L}-c_{R}=48 \pi K
$$

is insensitive to the detailed structure of the higher derivative terms and is determined completely by the coefficient of the Chern-Simons term. In the analysis of [9] this was a consequence of the fact that $c_{L}-c_{R}$ is related to the diffeomorphism anomaly of the bulk theory. In the present context this is a consequence of the fact that $c_{L}-c_{R}$ is determined by the parity odd part of the action evaluated on the near horizon geometry of the BTZ black hole, and this contribution comes solely from the Chern-Simons term.

\section{Non-extremal BTZ Black Holes}

We now turn to the computation of the entropy of a general non-extremal BTZ black hole solution given in eqs.(2.10), (2.11). First we note that the local geometry of extremal and non-extremal black holes are identical since both describe a locally $A d S_{3}$ space-time of curvature radius $l$. Thus $l$ for a non-extremal black hole is determined by the same equation as in the extremal case, 1.e. via the extremization of the function $g(l)$ :

$$
g^{\prime}(l)=0
$$

Since the contribution to the Noether charge from different terms in the action add, the entropy computed from Wald's general formula $[5,6,7,8]$ can be regarded as the sum of two terms, - one arising from the $\mathcal{L}_{0}^{(3)}$ term in the action and the other arising out of the $\mathcal{L}_{1}^{(3)}$ term in the action. In the $\rho-\tau-y$ coordinate system the contribution from the $\mathcal{L}_{0}^{(3)}$ 
term may be expressed as:

$$
\mathcal{E}_{0}=\left.8 \pi \int d y \sqrt{G_{y y}} \frac{\partial \mathcal{L}_{0}^{(3)}}{\partial \mathcal{R}_{\rho \tau \rho \tau}} G_{\rho \rho} G_{\tau \tau}\right|_{\rho=\rho_{+}}
$$

Here $\mathcal{R}_{M N P Q}$ denotes the Riemann tensor computed using the three dimensional metric $G_{M N}$ and in computing $\partial \mathcal{L}_{0}^{(3)} / \partial \mathcal{R}_{M N P Q}$ we need to treat $G_{M N}$ and $\mathcal{R}_{M N P Q}$ as independent variables. In writing (4.2) we have used the fact that all terms involving covariant derivatives of the Riemann tensor vanish in the BTZ black hole solution. Using the fact that in three dimensions $\mathcal{R}_{M N P Q}$ can be expressed in terms of $\mathcal{R}_{M N}$ and $G_{M N}$, and that for BTZ black hole both $\partial \mathcal{L}_{0}^{(3)} / \partial \mathcal{R}_{M N P Q}$ and $\mathcal{R}_{M N P Q}$ are proportional to $\left(G_{M P} G_{N Q}-G_{M Q} G_{N P}\right)$, (4.2) may be rewritten as[4]:

$$
\mathcal{E}_{0}=\left.\frac{4 \pi}{3}\left[\int d y \sqrt{G_{y y}} G_{M N} \frac{\partial \mathcal{L}_{0}^{(3)}}{\partial \mathcal{R}_{M N}}\right]\right|_{\rho=\rho_{+}}=\left.\frac{8 \pi^{2}}{3} \rho_{+}\left[G_{M N} \frac{\partial \mathcal{L}_{0}^{(3)}}{\partial \mathcal{R}_{M N}}\right]\right|_{\rho=\rho_{+}} .
$$

In order to evaluate the right hand side of (4.3) we note that for the BTZ black hole solution given in eq.(2.10),

$$
\mathcal{R}_{M N}=-2 l^{-2} G_{M N},
$$

and $\mathcal{L}_{0}^{(3)}=h(l)$ according to eq.(3.12). Thus

$$
G_{M N} \frac{\partial \mathcal{L}_{0}^{(3)}}{\partial \mathcal{R}_{M N}}=-\frac{1}{2} l^{2} \mathcal{R}_{M N} \frac{\partial \mathcal{L}_{0}^{(3)}}{\partial \mathcal{R}_{M N}}=-\frac{1}{2} l^{2} l^{-2} \frac{\partial}{\partial\left(l^{-2}\right)} h(l)=\frac{l^{3}}{4} h^{\prime}(l) .
$$

Using (3.15) this can be written as

$$
G_{M N} \frac{\partial \mathcal{L}_{0}^{(3)}}{\partial \mathcal{R}_{M N}}=-\frac{3}{\pi l} g(l)+\frac{1}{\pi} g^{\prime}(l)=\frac{3 C}{l},
$$

where in the second step we have used eqs.(3.17) and (4.1). Hence (4.3) gives

$$
\mathcal{E}_{0}=8 \pi^{2} C l^{-1} \rho_{+}
$$

Let us now turn to the contribution $\mathcal{E}_{1}$ from the Chern-Simons term. For this we shall view the black hole as a two dimensional solution and apply Wald's formula. This gives

$$
\mathcal{E}_{1}=8 \pi \frac{\partial \mathcal{L}_{1}^{(2)}}{\partial R_{\rho \tau \rho \tau}} g_{\rho \rho} g_{\tau \tau}=4 K \pi^{2} \frac{\varepsilon^{\mu \nu} F_{\mu \nu}}{\sqrt{-\operatorname{det} g}} \frac{1}{2} g^{\rho \rho} g^{\tau \tau} g_{\rho \rho} g_{\tau \tau}=-8 \pi^{2} K l^{-1} \rho_{-} .
$$


Thus the total entropy is

$$
\mathcal{E}=\mathcal{E}_{0}+\mathcal{E}_{1}=4 \pi^{2} l^{-1}\left((C-K)\left(\rho_{+}+\rho_{-}\right)+(C+K)\left(\rho_{+}-\rho_{-}\right)\right) .
$$

In order to express this as a function of the physical mass $M$ and angular momentum $J$ we need to relate $M$ and $J$ to the parameters $l$ and $\rho_{ \pm}$. We define $M$ and $J$ as the conserved Noether charges associated with time and $y$ translation symmetries respectively. The contribution splits into a sum of two terms, one coming from the $\mathcal{L}_{0}^{(3)}$ part and the other coming from the $\mathcal{L}_{1}^{(3)}$ part. The contribution from the $\mathcal{L}_{0}^{(3)}$ part was evaluated in [4] and is given by,

$$
M_{0} \pm J_{0}=\frac{2 \pi}{3 l}\left(\rho_{+} \pm \rho_{-}\right)^{2} G_{\mu \nu} \frac{\partial \mathcal{L}_{0}^{(3)}}{\partial R_{\mu \nu}}=\frac{2 \pi C}{l^{2}}\left(\rho_{+} \pm \rho_{-}\right)^{2},
$$

where in the second step we have used (4.6). On the other hand the contributions from $\mathcal{L}_{1}^{(3)}$ were computed in $[10,12]$ and are given by:

$$
J_{1}=-\frac{2 \pi K}{l^{2}}\left(\rho_{+}^{2}+\rho_{-}^{2}\right), \quad M_{1}=-\frac{4 \pi K}{l^{2}} \rho_{+} \rho_{-} .
$$

Eqs.(4.10) and (4.11) now give

$$
M \pm J=\left(M_{0}+M_{1}\right) \pm\left(J_{0}+J_{1}\right)=\frac{2 \pi(C \mp K)}{l^{2}}\left(\rho_{+} \pm \rho_{-}\right)^{2} .
$$

Substituting this into (4.9) and using the definitions of $c_{L}, c_{R}$ given in (3.21) we get

$$
\mathcal{E}=2 \pi \sqrt{\frac{c_{L} q_{L}}{6}}+2 \pi \sqrt{\frac{c_{R} q_{R}}{6}}
$$

where

$$
q_{L}=\frac{1}{2}(M-J), \quad q_{R}=\frac{1}{2}(M+J) .
$$

This is the correct expression for the entropy of a non-extremal black hole in the presence of higher derivative and Chern-Simons terms[9].

Note that the entropy and the near horizon geometry of the non-extremal BTZ black hole is determined by extremizing the function

$$
\mathcal{E}=2 \pi\left[q_{L} e_{L}+q_{R} e_{R}-\left(\frac{1}{e_{L}}+\frac{1}{e_{R}}\right) g(l)+\pi K\left(\frac{1}{e_{L}}-\frac{1}{e_{R}}\right)\right],
$$

with respect to $l, e_{L}, e_{R}$. Here

$$
e_{L}=\frac{l}{\rho_{+}-\rho_{-}}, \quad e_{R}=\frac{l}{\rho_{+}+\rho_{-}} .
$$

We suspect that one can follow the procedure of [15] to manipulate Wald's formula and the equations of motion to derive this extremization principle directly, but we have not so far succeeded in doing this. 


\section{References}

[1] M. Banados, C. Teitelboim and J. Zanelli, Phys. Rev. Lett. 69, 1849 (1992) [arXiv:hep-th/9204099].

[2] A. Strominger, JHEP 9802, 009 (1998) [arXiv:hep-th/9712251].

[3] J. D. Brown and M. Henneaux, Commun. Math. Phys. 104, 207 (1986).

[4] H. Saida and J. Soda, Phys. Lett. B 471, 358 (2000) [arXiv:gr-qc/9909061].

[5] R. M. Wald, Phys. Rev. D 48, 3427 (1993) [arXiv:gr-qc/9307038].

[6] T. Jacobson, G. Kang and R. C. Myers, Phys. Rev. D 49, 6587 (1994) [arXiv:grqc/9312023].

[7] V. Iyer and R. M. Wald, Phys. Rev. D 50, 846 (1994) [arXiv:gr-qc/9403028].

[8] T. Jacobson, G. Kang and R. C. Myers, arXiv:gr-qc/9502009.

[9] P. Kraus and F. Larsen, arXiv:hep-th/0506176.

[10] P. Kraus and F. Larsen, arXiv:hep-th/0508218.

[11] J. M. Maldacena and A. Strominger, JHEP 9812, 005 (1998) [arXiv:hep-th/9804085].

[12] S. N. Solodukhin, arXiv:hep-th/0509148.

[13] A. Achucarro and M. E. Ortiz, Phys. Rev. D 48, 3600 (1993) [arXiv:hep-th/9304068].

[14] G. Guralnik, A. Iorio, R. Jackiw and S. Y. Pi, Annals Phys. 308, 222 (2003) [arXiv:hep-th/0305117].

[15] A. Sen, JHEP 0509, 038 (2005) [arXiv:hep-th/0506177].

[16] A. Sen, arXiv:hep-th/0508042. 